\title{
Efficacy of transversus abdominis plane block in pain control after general abdominal surgery - a short review of the literature
}

\author{
Mihaela Butiulca*, Alexandra Lazăr \\ George Emil Palade University of Medicine, Pharmacy, Science, and Technology of Targu Mures, Romania
}

Objective: The aim of the study is the assessment of the analgesic efficacy of transversus abdominis plane block in patients undergoing general abdominal surgery. Methods: Pubmed, Scopus, and Medline databases were searched for papers evaluating the effect of transversus abdominis plane block. The primary and secondary outcomes of the studies were analyzed. Results: A total of 10 studies were analyzed, including 717 patients. Studies revealed that transversus abdominis plane block was associated with significantly reduced postoperative discomfort and reduced opioid consumption. Conclusion: The present study shows the clear benefit brought by the transversus abdominis plane block as part of multimodal analgesia, with a significant reduction of pain and higher comfort scores.

Keywords: transversus abdominis plane block, abdominal surgery, postoperative pain management, analgesic efficacy

Received 20 July 2021 / Accepted 11 September 2021

\section{Introduction}

Pain after surgery is a crucial variable in the recovery process of surgical patients, with a significant impact on the transition from acute to chronic pain [1,2]. Conventional postoperative analgesia proposes the use of opioids, efficient drugs that come with a series of adverse reactions such as nausea and vomiting, pruritus, sedation, constipation, and respiratory distress [3]. The inability to ensure efficient pain relief leads to undesirable complications and poor quality of life [4]. Safe and adequate preoperative analgesia is essential to improving post-procedure recovery [5]. Optimal analgesia aims to enhance patient comfort while minimizing the risk of complications, which may jeopardize the recovery process [6].

The available techniques for perioperative analgesia are neuraxial analgesia, patient-controlled analgesia with systemic opioids, or peripheral nerve blocks analgesia. The latest guidelines of the American Society of Anesthesiologists recommend the use of multimodal analgesia whenever possible. This involves the administration of two or more active substances, which act through different mechanisms to ensure efficient analgesia. They can be administered on the same route or by various means. Systemic administration of Acetaminophen, non-steroidal anti-inflammatory drugs like coxibs, are recommended in association with a regional analgesic technique for optimal pain management [7].

Surgery is an indispensable part of healthcare. Despite large benefits on life quality and survival, surgical procedures often result in acute pain. Abdominal surgery generates intense pain as a result of the large incision practiced in laparotomies, or pneumoperitoneum in laparoscopy [8].

* Correspondence to: Mihaela Butiulca

E-mail: mihaelabutiulca@gmail.com
Regional anesthesia techniques are significant components of efficient multimodal analgesia including, epidural anesthesia and peripheral nerve blocks [9]. For a long time, epidural anesthesia was considered the gold standard in abdominal surgery [10]. Epidural anesthesia is associated with numerous complications, including hypotension secondary to the sympathetic blockade, increased frequency of nausea and vomiting, and urinary retention. Therefore, experts in the field started to look for alternative analgesic techniques $[11,12]$.

The transversus abdominis plane block was first described in 2001 by Rafi [13]. This author described a technique based on anatomical landmarks, at the level of the Petit triangle, traversing with the needle the fascial plane between the transverse abdominal muscle and the internal oblique muscle and injecting local anesthetic at this level. This technique is based on the perception of a double loss of resistance resulting from the penetration of the two fascial planes. They managed to provide effective analgesia, comparable to epidural anesthesia, in the lower abdominal area, by blocking the thoracolumbar T6-L1 nerves [14]. In 2007 Hebbard first described the possibility of ultrasoundguided transversus abdominis plane block [15], thus improving the chances of success and significantly reducing the complications associated with the infiltration of local anesthetics in inappropriate regions. Ultrasonography is the method by which anatomical regions can be visualized with the help of ultrasound. This approach is widely accessible and implies low costs. Visualization of the anatomical structures limits the complications of these analgesic maneuvers. This method requires ultrasound scanning of the abdominal region on the median axillary line from the costal margin to the iliac crest. The three hypoechoic muscular layers, the external oblique muscle, the internal oblique muscle, and the transversus abdominis muscle, are separated by the 
muscular fasciae, with hyperechoic aspect. Local anesthetic is injected between the fascia of the internal oblique muscle and the fascia of the transversus abdominis muscle. Proper injection of the anesthetic resembles a "goose egg" shape if the technique is performed correctly [16].

Indications for transversus abdominis plane block are mainly subumbilical surgical procedures, but through a subcostal approach, analgesia can be achieved for supraumbilical surgical procedures, such as laparoscopy [17]. Contraindications of this procedure can be absolute or relative. The patient's refusal, allergy to the local anesthetic substance, the existence of a localized infection at the site of injection represent absolute contraindications. Relative contraindications include coagulopathy and abdominal wall abnormalities due to previous surgical procedures (hernias, diastasis) [18].

This review aims to underline the benefit brought by the transversus abdominis plane block as part of multimodal analgesia following abdominal surgery.

\section{Material and methods}

The presented work is a review of the literature which analyses the efficiency of the transverse abdominal plane block. We used the databases Pubmed, Scopus, and Medline, using the terms "TAP block" or "transversus abdominis plane block" or "TAP" or "ultrasound-guided regional anesthesia" or "abdominal wall block". The search strategy was limited to studies published in the last five years, human studies, randomized studies. All the studies analyzed were published in English.

The present paper includes patients aged between 18 to 85 years old, both men and women, who underwent abdominal surgical interventions. Only studies that describe bilateral abdominal wall block under ultrasound guidance were accepted, regardless of the abdominal surgical technique performed.

Data was gathered regarding the surgical procedure, the type of local anesthetic used, and the results obtained from each study. We defined exclusion criteria as the following: unrandomized studies, studies with less than 60 patients, case reports, unilateral laparoscopic procedures, gynecologic and urological pathology.

\section{Results}

A survey of medical literature revealed 114 studies that corresponded to the keyword search. After the exclusion criteria were applied, 92 studies were rejected due to ineligibility. Finally, 10 studies remain eligible to be used in this review. A flow diagram of the entire review process is represented in Figure 1. The included studies were published between 2016 and 2020, cumulating a total of 717 patients. Detailed characteristics are available in Table I. Data gathering was done by a single person. All studies included in the present work are randomized, $40 \%(\mathrm{n}=4)$ double-blind, 40\% ( $\mathrm{n}=4)$ single-blind, and 20\% ( $\mathrm{n}=2)$ non-blind (Figure 2).

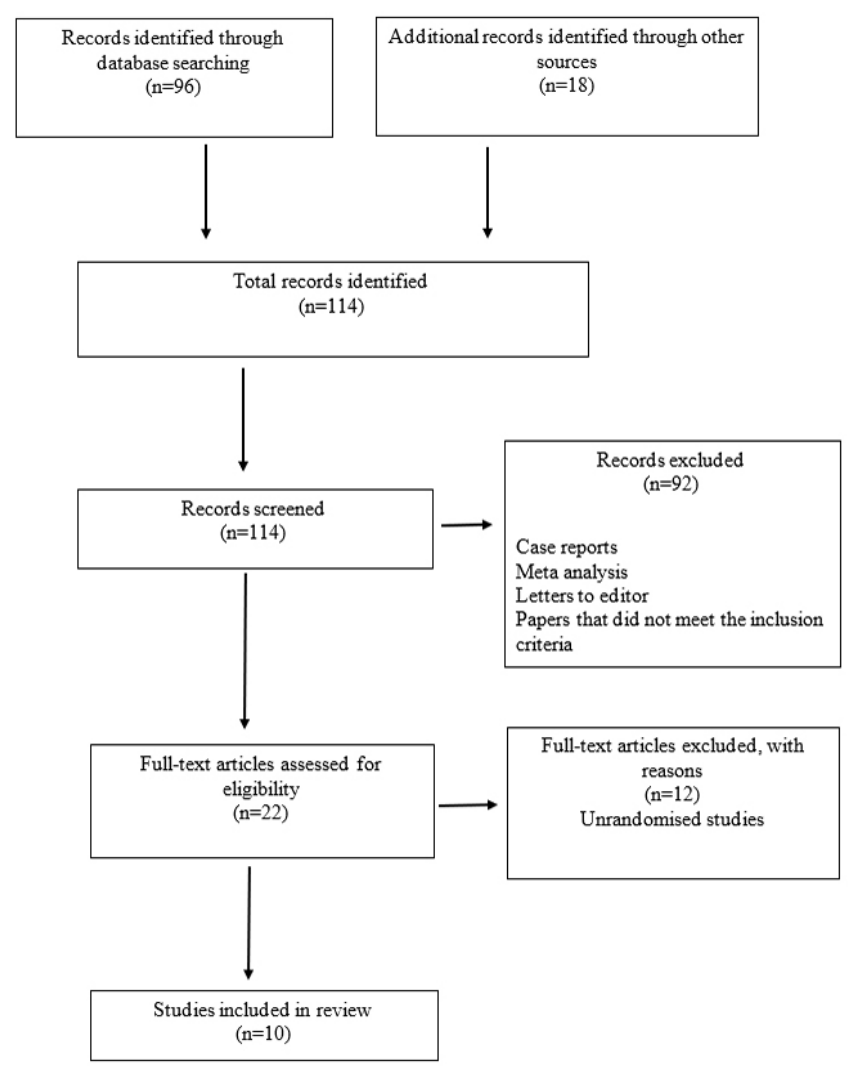

Fig. 1. Flow diagram showing the review process according to PRISMA Group(2009)[33]

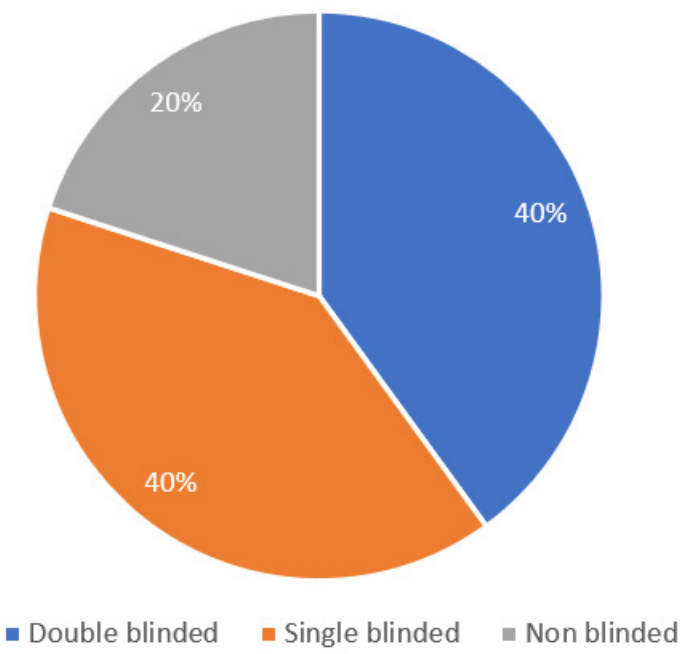

Fig. 2. The randomization of the analysed studies

The abdominal pathology in the analyzed papers was oncological in 70\% ( $\mathrm{n}=7)$ of studies, bariatric surgery in $20 \%$ $(\mathrm{n}=2)$, and bilateral inguinal hernia in 10\% $(\mathrm{n}=1)$ (Figure 3). Laparoscopy surgery was performed in $60 \%(\mathrm{n}=6)$ of studies, conventional surgery in $30 \%(\mathrm{n}=3)$, and one study did not specify the type of surgical technique used.

In $70 \%$ of trials, the transversus abdominis plane block was executed preoperatively, after the general anesthesia, $20 \%$ of the cases it was performed after surgery, and one study does not specify the moment of the transverse abdominal plane block. Two local anesthetics, Ropivacaine $(50 \%)$ and Bupivacaine $(50 \%)$, were used. The use of an- 

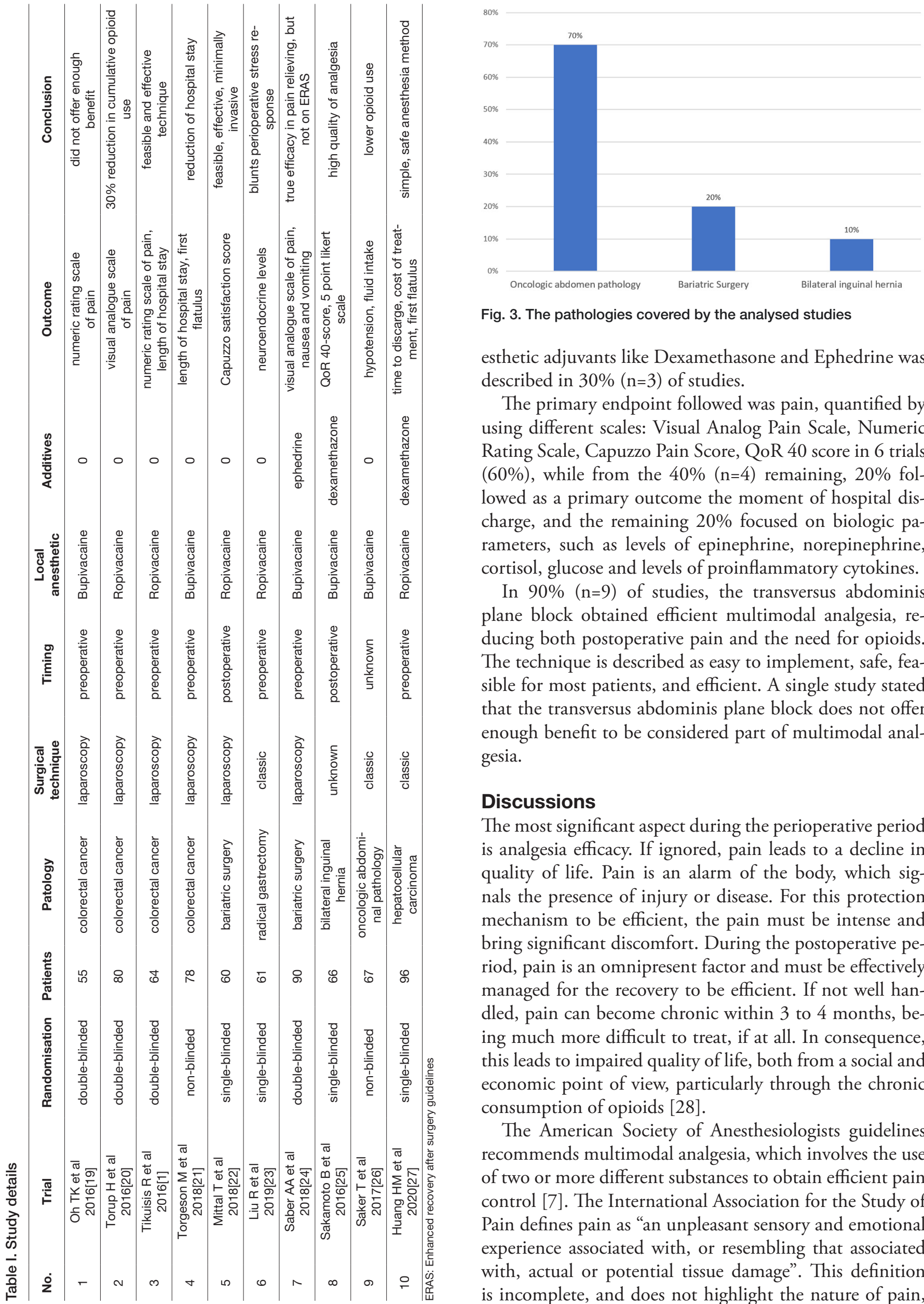

Fig. 3. The pathologies covered by the analysed studies

esthetic adjuvants like Dexamethasone and Ephedrine was described in 30\% ( $n=3)$ of studies.

The primary endpoint followed was pain, quantified by using different scales: Visual Analog Pain Scale, Numeric Rating Scale, Capuzzo Pain Score, QoR 40 score in 6 trials $(60 \%)$, while from the $40 \%(\mathrm{n}=4)$ remaining, $20 \%$ followed as a primary outcome the moment of hospital discharge, and the remaining $20 \%$ focused on biologic parameters, such as levels of epinephrine, norepinephrine, cortisol, glucose and levels of proinflammatory cytokines.

In $90 \%(n=9)$ of studies, the transversus abdominis plane block obtained efficient multimodal analgesia, reducing both postoperative pain and the need for opioids. The technique is described as easy to implement, safe, feasible for most patients, and efficient. A single study stated that the transversus abdominis plane block does not offer enough benefit to be considered part of multimodal analgesia.

\section{Discussions}

The most significant aspect during the perioperative period is analgesia efficacy. If ignored, pain leads to a decline in quality of life. Pain is an alarm of the body, which signals the presence of injury or disease. For this protection mechanism to be efficient, the pain must be intense and bring significant discomfort. During the postoperative period, pain is an omnipresent factor and must be effectively managed for the recovery to be efficient. If not well handled, pain can become chronic within 3 to 4 months, being much more difficult to treat, if at all. In consequence, this leads to impaired quality of life, both from a social and economic point of view, particularly through the chronic consumption of opioids [28].

The American Society of Anesthesiologists guidelines recommends multimodal analgesia, which involves the use of two or more different substances to obtain efficient pain control [7]. The International Association for the Study of Pain defines pain as "an unpleasant sensory and emotional experience associated with, or resembling that associated with, actual or potential tissue damage". This definition is incomplete, and does not highlight the nature of pain, 
or whether it is chronic or acute. The main problem is the assessment of pain, which is a subjective factor. Most frequently pain is quantified with the aid of a visual, verbal, or numerical pain scale. The sensitivity and confidence provided by these are low [29].

One of the studies described, Liu R et al. [23], tried to evaluate the systemic response to postoperative stress through a visual pain scale. They analyzed patients undergoing a radical gastrectomy for oncologic pathology, through classic surgical techniques. The study sample included 61 patients, divided into two groups: 30 patients who benefited from transversus abdominis plane block and 31 patients in the control group. The primary outcome of the study was the assessment of norepinephrine, epinephrine, C-reactive protein, cortisol, glucose, interleukins 6 and 10 levels. The groups were homogeneously divided, so the basal values of the neuroendocrine mediators and the proinflammatory cytokines were similar in both groups. Noradrenaline, epinephrine, and cortisol values were significantly lower and more stable in the transversus abdominis plane block group, compared with the control group, and returned to basal values within 24 hours. Similarly, glucose values were lower in the transversus abdominis plane block group compared with the control group. Inflammatory cytokines continued to rise in the placebo group, even 48 hours after surgery, while in the transversus abdominis plane block group they started to fall after 24 hours. The biologic parameters were stable during the surgery in patients who benefited from the abdominal block. The postoperative drug consumption, both opioids, and nonopioids drugs were also lower for these patients. Another parameter analyzed was the pain scores on the visual pain scale, with no significant differences between the two groups. The study concluded that this analgesic technique could reduce body stress levels periprocedural, and it could provide satisfactory pain relief without further complications. This work emphasizes the role of preemptive analgesia. Although the satisfaction level of patients in the two groups was similar, the clinical and paraclinical elements contradict these findings, showing clearly that the transversus abdominis plane block is superior to conventional analgesia. The difference between subjective pain levels and biological markers underlines the high variability when assessing pain, as the most significant obstacle. However, there is a more precise way to evaluate pain, using the help of functional brain magnetic resonance imaging, but this method is not widely available [30].

Mittal T et al.[22] and Sakamoto B et al. [25] use Capuzzo score and QoR 40-score for assessing the patient's comfort and analgesia, concluding that the transversal abdominal block is an effective technique with no underlying risk.

Length of hospital stay was another significant parameter followed by the authors, which was less in the target group, compared to the control group (Torgeson $\mathrm{M}$ et $\mathrm{al}[21]$ and Huang HM et al.[27].
In general, studies had patients divided into two groups, one that benefited from transversus abdominis plane block and another that either did not undergo an abdominal block or received transversus abdominis plane block with saline. These studies are essential since they emphasize the efficacy of transversus abdominis plane block used as a minimally invasive postoperative multimodal analgesia. Torgeson $\mathrm{M}$ et al. analyzed the benefits of performing a transversal plane block compared to conventional epidural anesthesia in patients undergoing colorectal surgery. The study included 83 patients subdivided into two groups: 39 patients in the epidural analgesia group and 44 patients in the abdominal block group. Time to first bowel movement was comparable in both groups. Length of hospital stay was significantly shorter in the patients who benefited from the transverse abdominal block by 0.5 days. Nausea and vomiting episodes were more frequent in the transversus abdominis plane block group, and urinary retention was more frequent in the epidural group. The study concluded that patients with prior postoperative urinary retention benefit the most from transversus abdominis plane block. The abdominal block is providing a feasible alternative for patients undergoing colorectal surgery. Despite the unpleasant episodes of nausea and vomiting postoperatively, all results show the benefit brought by transversus abdominis plane block compared to epidural anesthesia [21].

Enhanced recovery after surgery (ERAS) guidelines were created focusing on improving the patient's recovery after surgery. ERAS recommends a multimodal approach to reduce postoperative stress [32]. Huang HM et al. focused on the recovery of patients who have undergone elective liver resection for hepatocellular carcinoma. The authors of the study suggest the use of laryngeal mask airway to protect the respiratory tract during general anesthesia in addition to the transversus abdominis plane block. The study group consists of 96 patients subdivided into two groups of 48 patients each. Patients in the control group benefited from general anesthesia with endotracheal intubation, while the study group received general anesthesia with laryngeal mask airway and transversus abdominis plane block. The primary outcome measured was the time to discharge. Patients receiving transversus abdominis plane block were discharged on average one day earlier, compared to the control group. Patients in the laryngeal mask airway and transversus abdominis plane block group had significantly lower pain scores, mobilization off the bed was faster, and bowel movement returned sooner. There were no significant differences in nausea and vomiting, and the 40-point QoR satisfaction score was similar on the first day after surgery but significantly higher on day 3. Patients in the transversus abdominis plane block group also used fewer opioids during surgery and spent less time in the post-anesthesia care unit. The study concludes that the association between laryngeal mask airway and transversus abdominis plane block is safe and effective as part of the ERAS, it reduces postoperative stress and helps in the recovery process [27]. 
The present review suggests that the transversus abdominis plane block is associated with reduced opioid consumption, a statement supported by all the studies analyzed. But this decrease in opioid consumption is limited to the first 24 hours postoperatively, as ropivacaine and bupivacaine are both long-acting local anesthetics, but no longer than 15 to 20 hours [33]. Besides reducing the opioid after surgery, good management of postoperative pain decreases the risks of incisional hernias [34].

Although this review answers a few essential questions about the transverse abdominal block, it leaves some essential areas uncovered such as: what is the most appropriate time to perform the transversus abdominis plane block, which is the most appropriate anesthetic agent to use, and which is the optimal concentration, which is the most appropriate technique and anatomic region to be approached. But the most important question remains unanswered: what is the most optimal and efficient method to evaluate perioperative pain?

Limitations of the study were represented by the small number of studies evaluated, the limited number of patients in each study, and the heterogeneity of the studies addressed.

\section{Conclusions}

The present review shows the clear benefit brought by the transversus abdominis plane block as part of multimodal analgesia, with a significant reduction of pain and higher comfort scores. Also, other parameters such as length of hospital stay, mobilization off the bed, bowel movement, and urinary retentions were significantly improved compared with the control groups. Proper evaluation and precise management of perioperative pain syndromes are the challenges faced in this review article.

\section{Authors' contribution}

MB - substantial contribution to conception and design, acquisition and interpretation of data, drafting the article, final approval of the version to be published

LA - substantial contribution to conception and design, substantial contribution to revising the article critically for important intellectual content, final approval of the version to be published

\section{Conflict of interest}

None to declare.

\section{References}

1. Tikuisis R, Miliauskas $\mathrm{P}$, Lukoseviciene $\mathrm{V}$, et al. Transversus abdominis plane block for postoperative pain relief after hand-assisted laparoscopic colon surgery: a randomized, placebo-controlled clinical trial. Tech Coloproctol. 2016 Dec;20(12):835-844.

2. Glare P, Aubrey KR, Myles PS. Transition from acute to chronic pain after surgery. Lancet. 2019 Apr 13;393(10180):1537-1546.

3. Kehlet H, Rung GW, Callesen T. Postoperative opioid analgesia: time for a reconsideration? J Clin Anesth. 1996 Sep;8(6):441-

4. Hunter C, Shakir A, Momeni A, et al. Transversus Abdominis Plane Block and Free Flap Abdominal Tissue Breast Reconstruction: Is There a True Reduction in Postoperative Narcotic Use? Ann Plast Surg. 2017
Mar;78(3):254-259.

5. Rahiri J, Tuhoe J, Svirskis D, et al. Systematic review of the systemic concentrations of local anaesthetic after transversus abdominis plane block and rectus sheath block. Br J Anaesth. 2017 Apr 1;118(4):517526.

6. Andreae MH, Andreae DA. Local anaesthetics and regional anaesthesia for preventing chronic pain after surgery. Cochrane Database Syst Rev. 2012 Oct 17;10:CD007105.

7. American Society of Anesthesiologists Task Force on Acute Pain Management. Practice guidelines for acute pain management in the perioperative setting: an updated report by the American Society of Anesthesiologists Task Force on Acute Pain Management. Anesthesiology. 2012 Feb;116(2):248-73.

8. Novak-Jankovič V, Markovič-Božič J. Regional anaesthesia in thoracic and abdominal surgery. Acta Clin Croat. 2019 Jun;58(Suppl 1):96-100.

9. Urits I, Ostling PS, Novitch MB, et al. Truncal regional nerve blocks in clinical anesthesia practice. Best Pract Res Clin Anaesthesiol. 2019 Dec;33(4):559-571.

10. Rodgers A, Walker N, Schug S, et al. Reduction of postoperative mortality and morbidity with epidural or spinal anaesthesia: results from overview of randomised trials. BMJ. 2000 Dec 16;321(7275):1493.

11. Pöpping DM, Elia N, Van Aken HK, et al. Impact of epidural analgesia on mortality and morbidity after surgery: systematic review and meta-analysis of randomized controlled trials. Ann Surg. 2014 Jun;259(6):1056-67.

12. Bos EME, Hollmann MW, Lirk P. Safety and efficacy of epidural analgesia. Curr Opin Anaesthesiol. 2017 Dec;30(6):736-742.

13. Rafi AN. Abdominal field block: a new approach via the lumbar triangle. Anaesthesia. 2001 Oct;56(10):1024-6.

14. Hamid HKS, Ahmed AY, Saber AA, Emile SH, Ibrahim M, Ruiz-Tovar J. Transversus abdominis plane block using a short-acting local anesthetic reduces pain and opioid consumption after laparoscopic bariatric surgery: a meta-analysis. Surg Obes Relat Dis. 2020 Sep;16(9):13491357.

15. Hebbard P, Fujiwara $Y$, Shibata $Y$, Royse C. Ultrasound-guided transversus abdominis plane (TAP) block. Anaesth Intensive Care. 2007 Aug;35(4):616-7.

16. Keller DS, Madhoun N, Ponte-Moreno OI, Ibarra S, Haas EM. Transversus abdominis plane blocks: pilot of feasibility and the learning curve. J Surg Res. 2016 Jul;204(1):101-8.

17. Sinha A, Jayaraman L, Punhani D, Chowbey P. Transversus abdominis plane block for pain relief in patients undergoing in endoscopic repair of abdominal wall hernia: A comparative, randomised double-blind prospective study. J Minim Access Surg. 2018 Jul-Sep;14(3):197-201.

18. Ashken T, West $\mathrm{S}$. Regional anaesthesia in patients at risk of bleeding. BJA Educ. 2021 Mar;21(3):84-94.

19. Oh TK, Yim J, Kim J, et al. Effects of preoperative ultrasound-guided transversus abdominis plane block on pain after laparoscopic surgery for colorectal cancer: a double-blind randomized controlled trial. Surg Endosc. 2017 Jan;31(1):127-134.

20. Torup $H$, Hansen EG, Bøgeskov M, et al. Transversus abdominis plane block after laparoscopic colonic resection in cancer patients: A randomised clinical trial. Eur J Anaesthesiol. 2016 Oct;33(10):725-30.

21. Torgeson M, Kileny J, Pfeifer C, Narkiewicz L, Obi S. Conventional Epidural vs Transversus Abdominis Plane Block with Liposomal Bupivacaine: A Randomized Trial in Colorectal Surgery. J Am Coll Surg. 2018 Jul;227(1):78-83.

22. Mittal T, Dey A, Siddhartha R, Nali A, Sharma B, Malik V. Efficacy of ultrasound-guided transversus abdominis plane (TAP) block for postoperative analgesia in laparoscopic gastric sleeve resection: a randomized single blinded case control study. Surg Endosc. 2018 Dec;32(12):4985-4989.

23. Liu R, Qin H, Wang M, Li K, Zhao G. Transversus abdominis plane block with general anesthesia blunts the perioperative stress response in patients undergoing radical gastrectomy. BMC Anesthesiol. 2019 Nov 7;19(1):205.

24. Saber AA, Lee YC, Chandrasekaran A, et al. Efficacy of transversus abdominis plane (TAP) block in pain management after laparoscopic sleeve gastrectomy (LSG): A double-blind randomized controlled trial. Am J Surg. 2019 Jan;217(1):126-132.

25. Sakamoto B, Harker G, et al. Efficacy of Local Anesthetic With Dexamethasone on the Quality of Recovery Following Total Extraperitoneal Bilateral Inguinal Hernia Repair: A Randomized Clinical Trial. JAMA Surg. 2016 Dec 1;151(12):1108-1114.

26. Shaker TM, Carroll JT, Chung MH, et al. Efficacy and safety of transversus abdominis plane blocks versus thoracic epidural anesthesia in patients undergoing major abdominal oncologic resections: A prospective, 
randomized controlled trial. Am J Surg. 2018 Mar;215(3):498-501.

27. Huang HM, Chen RX, Zhu LM, et al. Combined use of transversus abdominis plane block and laryngeal mask airway during implementing ERAS programs for patients with primary liver cancer: a randomized controlled trial. Sci Rep. 2020 Sep 10;10(1):14892.

28. Hyland SJ, Brockhaus KK, Vincent WR, et al. Perioperative Pain Management and Opioid Stewardship: A Practical Guide. Healthcare (Basel). 2021 Mar 16;9(3):333.

29. Tracey I, Woolf CJ, Andrews NA. Composite Pain Biomarker Signatures for Objective Assessment and Effective Treatment. Neuron. 2019 Mar 6;101(5):783-800.

30. Morton DL, Sandhu JS, Jones AK. Brain imaging of pain: state of the art. J Pain Res. 2016;9:613-624.

31. Melloul, E., Hübner, M., Scott, M, et al. Guidelines for Perioperative Care for Liver Surgery: Enhanced Recovery After Surgery (ERAS) Society Recommendations. World J Surg 2016 Nov 40(11): 2425-2440.

32. Cuvillon P, Nouvellon E, Ripart J, et al. A comparison of the pharmacodynamics and pharmacokinetics of bupivacaine, ropivacaine (with epinephrine) and their equal volume mixtures with lidocaine used for femoral and sciatic nerve blocks: a double-blind randomized study. Anesth Analg. 2009 Feb;108(2):641-9.

33. Moher D, Liberati A, Tetzlaff J, Altman DG, The PRISMA Group (2009). Preferred Reporting Items for Systematic Reviews and Meta-Analyses: The PRISMA Statement. PLoS Med 6(7).

34. Gherghinescu M, Copotoiu C, Lazar A, Popa D, Mogoanta SS, Molnar C. Continuous local analgesia is effective in postoperative pain treatment after medium and large incisional hernia repair. Hernia. 2017 Oct;21(5):677-685. 